\title{
Evaluation of wearable haptic systems for the fingers in Augmented Reality applications
}

\author{
Maurizio Maisto, Claudio Pacchierotti, Francesco Chinello, Gionata Salvietti, \\ Alessandro De Luca, Domenico Prattichizzo
}

\begin{abstract}
Although Augmented Reality (AR) has been around for almost five decades, only recently we have witnessed AR systems and applications entering in our everyday life. Representative examples of this technological revolution are the smartphone games "Pokémon GO" and "Ingress" or the Google Translate real-time sign interpretation app. Even if AR applications are already quite compelling and widespread, users are still not able to physically interact with the computer-generated reality. In this respect, wearable haptics can provide the compelling illusion of touching the superimposed virtual objects without constraining the motion or the workspace of the user. In this paper, we present the experimental evaluation of two wearable haptic interfaces for the fingers in three AR scenarios, enrolling 38 participants. In the first experiment, subjects were requested to write on a virtual board using a real chalk. The haptic devices provided the interaction forces between the chalk and the board. In the second experiment, subjects were asked to pick and place virtual and real objects. The haptic devices provided the interaction forces due to the weight of the virtual objects. In the third experiment, subjects were asked to balance a virtual sphere on a real cardboard. The haptic devices provided the interaction forces due to the weight of the virtual sphere rolling on the cardboard. Providing haptic feedback through the considered wearable device significantly improved the performance of all the considered tasks. Moreover, subjects significantly preferred conditions providing wearable haptic feedback.
\end{abstract}

Index Terms - wearable haptics, augmented reality, AR, cutaneous feedback, tactile feedback, fingertip haptics

\section{INTRODUCTION}

A UGMENTED Reality (AR) enables to supplement the realworld environment with computer-generated sensory inputs, such as virtual objects, animations, and sounds. AR systems have been around for already 50 years [1], but only the recent development and commercialization of inexpensive and portable computers are bringing AR applications in our everyday life. In this respect, a notable example is the Google Glass, a futuristic idea for virtual and augmented reality hosted in a pair of eye glasses: video calls, pictures, maps, and many other types of visual information, are available at a single glance, superimposed to the real world. More recently, AR made the headlines with the smartphone game "Pokémon GO", which renders virtual animated creatures on top of the real world. The application uses the smartphone camera to capture the surrounding environment, to which it adds the fictional pocket monsters. Users can swipe on the screen to throw a virtual "Poké Ball" to the creature and capture it. After less than 1 month from its release, the "Pokémon GO" application had been downloaded more than 75 million times [2]. And this success seems only the very first step toward a bright and popular

- M. Maisto and A. De Luca are with the Dipartimento di Ingegneria Informatica, Automatica e Gestionale, Sapienza Universita di Roma, Rome, Italy. (e-mail: maurizio.maisto1992@gmail.com; deluca@diag.uniroma1.it).

- C. Pacchierotti is with the CNRS at Irisa and Inria Rennes Bretagne Atlantique, Rennes, France. E-mail: claudio.pacchierotti@irisa.fr.

- F. Chinello, G. Salvietti, and D. Prattichizzo are with the Dept. of Advanced Robotics, Istituto Italiano di Tecnologia, Genova, Italy. E-mail: \{francesco.chinello, gionata.salvietti, domenico.prattichizzo\}@iit.it.

- G. Salvietti and D. Prattichizzo are also with the Dept. of Information Engineering and Mathematics, University of Siena, Siena, Italy.

- This research has received funding from the European Union Seventh Framework Programme FP7/2007-2013 under grant $n^{\circ} 601165$ of the project "WEARHAP - WEARable HAPtics for humans and robots". future for AR: Apple is reported to be "pouring money into [...] augmented reality [3]", Facebook is "researching AR very seriously [4]", Google is "working on a high-end standalone headset - one that mixes features of augmented reality and virtual reality [5]", and Microsoft expects "80 million mixed reality devices to be sold by 2020" [6].

Although AR applications are already quite enthralling, users are still not able to physically interact with the computergenerated inputs. We can throw Poké Balls to the virtual creatures, but we cannot pet them. This is mainly due to the lack of suitable interfaces, which should be capable of providing compelling sensations and, at the same time, be comfortable to wear, small, lightweight, and inexpensive. Wearable haptic interfaces are up for this challenge. In the past years we have in fact seen a great advancement of wearable haptics technologies. Notable commercial examples are the Google Moto 360, the Asus ZenWatch, the Samsung Gear Live, and the Apple Watch. They are as easy and comfortable to wear as a watch, they often features a touch screen, and they have functions similar to smartphones. Google and Apple even developed dedicated operating systems, which provide functions and applications customized for their wearable devices. However, the haptic stimuli provided by these wearables are still limited to vibrations, reducing the possibility of simulating rich contact interactions. For this reason, researchers have recently focused on more complex haptic interfaces, able to convey richer tactile sensations while showing compact form factors and light weights. For example, Solazzi et al. [7] developed a 3-DoF wearable cutaneous display for the fingertip: two degrees of freedom for the orientation and one linear degree of freedom to control the contact force. The motors are placed on the forearm and two cables for each actuated finger transmit the motor 


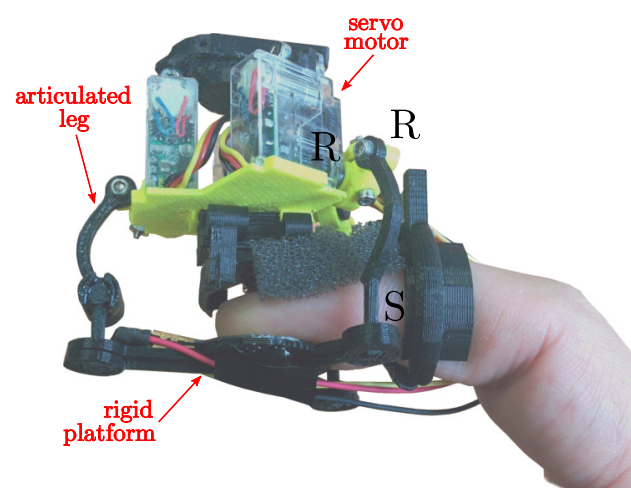

(a) 3-RRS fingertip device (side view, worn on the index finger).

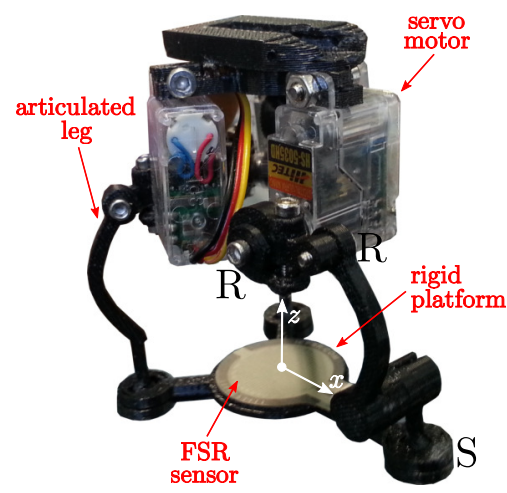

(b) 3-RRS fingertip device (top view).

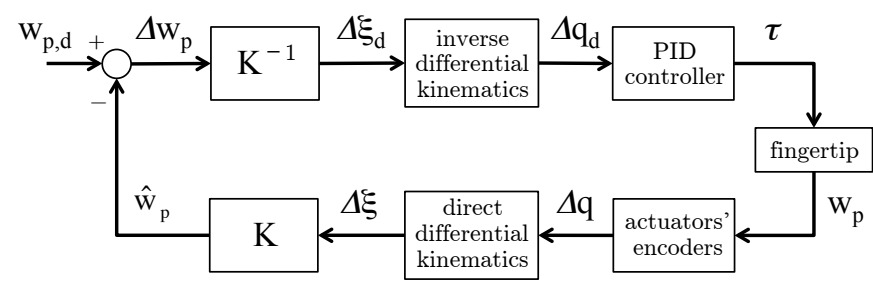

(c) 3-RRS fingertip device control scheme.

Fig. 1. The 3-RRS fingertip device. A rigid platform, driven by three servo motors, provides 3-DoF contact deformation stimuli to the user fingertip Each leg, connecting the end-effector to the upper body, is composed of two rigid links connected to each other, the body, and the end-effector, according to a RRS (Revolute-Revolute-Spherical) kinematic chain.

torque. Gabardi et al. [8] improved this fingertip device by replacing sheathed tendons actuation with DC motors mounted directly on the joints. Moreover, they increased the portability and wearability of the system by reducing the overall weight and dimensions. The total weight of this device is $30 \mathrm{~g}$ for $66 \times 35 \times 38 \mathrm{~mm}$ dimensions. Prattichizzo et al. [9] presented a wearable 3-DoF cutaneous device for interaction with virtual and remote environments. It consists of two platforms: one is located on the back of the finger, supporting three small DC motors, and the other is in contact with the volar surface of the fingertip. The motors shorten and lengthen three cables to move the platform toward the user's fingertip and re-angle it to simulate contacts with arbitrarily oriented surfaces. The direction and amount of the force reflected to the user is changed by properly controlling the cable lengths. Three force-sensing resistors near the platform vertices measure the fingertip contact force for closed-loop control. Koo et al. [10] addressed the wearability

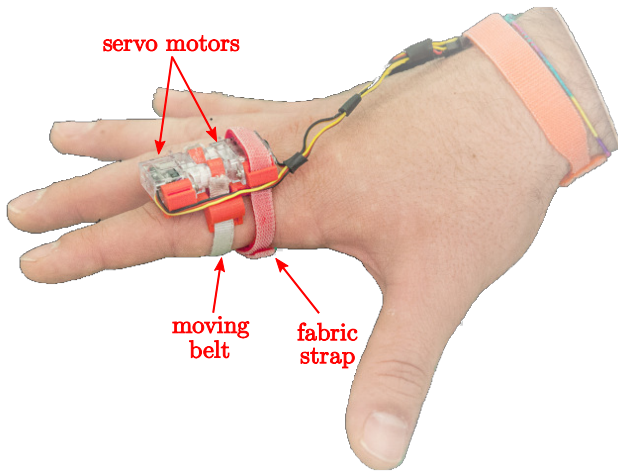

(a) hRing finger device.

Motors spin in opposite Motors spin in the same directions generating direction generating normal forces

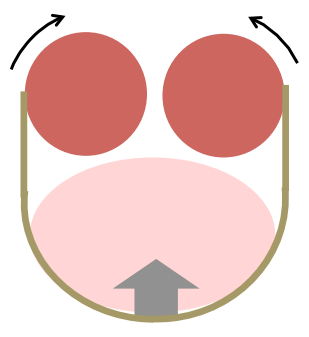
shear forces

(b) hRing actuation principle.

Fig. 2. The hRing finger device. A moving belt, driven by two servo motors, provides skin stretch and normal stimuli to the finger skin.

challenge of fingertip devices by using dielectric elastomer actuators, that can provide cutaneus stimuli without any electromechanical transmission. Their device is composed of a $4 \times 5$ array of stimulating cells. The total active area for the device is $11 \times 14 \mathrm{~mm}$, and the centers of tactile stimulating elements are $3 \mathrm{~mm}$ apart. Each element is $2 \mathrm{~mm}$ in diameter, the initial height is $0.1 \mathrm{~mm}$, and the maximum displacement is $0.45 \mathrm{~mm}$. The entire device is flexible and lightweight like a bandage. Similarly, Frediani et al. [11] described a wearable wireless cutaneous display, able to mechanically stimulate the fingertip. The device is based on dielectric elastomer actuators as well. The actuators are placed in contact with the finger pulp, inside a plastic case, which also hosted a compact high-voltage circuitry. A custom wireless control unit is fixed on the forearm and connected to the display via low-voltage leads. More recently, Leonardis et al. [12], [13] presented a 3RSR wearable skin stretch device for the fingertip. It moves a rigid tactor in contact with the skin, providing skin stretch and making/breaking contact sensations. An asymmetrical 3RSR configuration allows compact dimensions with minimum obstruction of the hand workspace and minimum inter-finger interference. Similarly, Girard et al. [14] developed a wearable haptic device able to simulate 2-DoF shear forces at the fingertip. It is composed of a parallelogram structure actuated by two DC motors that move a tactor in contact with the fingertip. It weights $22 \mathrm{~g}$ for a total dimension of $20 \times 34 \times 35 \mathrm{~mm}$. The tactor's maximum displacement is $2 \mathrm{~mm}$ in both directions.

Although these devices have been successfully employed in many applications and scenarios, they have all been 
designed to be worn on the fingertips. This is perfectly acceptable in virtual reality (VR) scenarios, where all the considered objects are virtual [14], [15], [16], [17], [18]. However, this may not be the best choice for AR applications. In fact, while in VR the user interacts only with virtual objects, in AR the user interacts with both virtual and real objects. For this reason, it seems a good idea to employ haptic devices that do not prevent the wearer from using their fingertips to interact with the real environment. In this respect, Arata et al. [19] presented a lightweight hand exoskeleton that left the palmar side of the hand almost free. The system is driven through large deformations of a compliant mechanism body, and it weights $320 \mathrm{~g}$. It is designed to distribute 1-DoF actuated linear motion into three rotational motions of the finger joints, which translate into natural finger flexion/extension. The portability of this exoskeleton has been later on improved by Nycz et al. [20] using a remote actuation system. A push-pull Bowden cable is used to transmit actuator forces from a backpack to the hand. This approach reduced the hand exoskeleton weight by over $50 \%$ without adverse effects to functionality. Uchiyama et al. [21] presented a vibrotactile glove providing directions and spatial representation to wheelchair users with severe visual impairment. The vibration signals are provided through a 3-by-3 array of vibrotactile actuators placed on the back of the hand. More recently, Hayes [22] provided vibrotactile feedback on the hand for hapticenabled music performances. She integrated two vibrotactile motors on the palm to recreate the vibrations produced by an acoustic instrument. The fingertips are left free to interact with the environment. Similar systems, featuring different arrangements of vibrotactile actuators across the hand, have shown promising results in various applications, such as robot-assisted surgery [23], guidance of visuallyimpaired people [24], virtual reality [25], [26], and enhanced cinematic experiences [27]. A detailed review of the literature on wearable haptics systems for the fingertip and the hand can be found in [28].

However, applications combining wearable haptics and $\mathrm{AR}$ are not common. One of the few examples is the work by Scheggi et al. [29], where a bi-manual experience involving wearable fingertip devices and an AR environment is presented. They used a grounded haptic interface and two wearable fingertip devices to provide, at the same time, haptic information about the shape and weight of a virtual object rendered in the real-world environment.

\subsection{Contribution}

This paper presents the evaluation of two wearable haptic systems for the fingers in three representative augmented reality applications. The first wearable device is a 3-RRS fingertip cutaneous device [30], shown in Fig. 1. A rigid platform, driven by three servo motors, provides 3-DoF contact deformation stimuli to the user fingertip. The second wearable device is a 2-DoF skin stretch cutaneous device [31], shown in Fig. 2. It is worn on the finger proximal phalanx and leaves the fingertip completely free. A moving belt, driven by two servo motors, provides skin stretch and normal stimuli to the finger skin.

We tested these two wearable interfaces in three AR scenarios. In the first experiment, subjects are requested to write on a virtual board using a real chalk. The haptic devices provide the interaction forces between the chalk and the board. In the second experiment, subjects are asked to pick and place virtual and real objects. The haptic devices provide the interaction forces due to the weight of the virtual objects being picked up. In the third experiment, subjects are asked to balance a virtual sphere on a real cardboard. The haptic devices provide the interaction forces due to the weight of the virtual sphere rolling on the cardboard.

Our hypothesis is that wearable haptic devices markedly improve the performance and illusion of presence of augmented reality environments, causing no significant impairment or discomfort to the users.

\section{Wearable cutaneous devices}

We tested our hypothesis using two of our wearable cutaneous devices. Below we summarize their features and present their force control approaches.

\subsection{3-RRS fingertip device}

Description. The 3-RRS fingertip device has been preliminarily presented by Chinello et al. [30], and it is shown in Fig. 1a. It is composed of a static upper body and a mobile end-effector. The upper body is located on the nail side of the finger, supporting three small servo motors, and the mobile end-effector is in contact with the finger pulp. The two parts are connected by three articulated legs, actuated by the motors. The end-effector can move toward the user's fingertip and rotate to simulate contacts with arbitrarily-oriented surfaces. A FSR 402 Short sensor (Interlink Electronics, USA) is placed on the platform to detect contact with the finger pulp. Each leg, connecting the end-effector to the upper body, is composed of two rigid links connected to each other, the body, and the endeffector, according to a RRS (Revolute-Revolute-Spherical) kinematic chain. Specifically, three spherical (S) joints connect the distal links of the legs to the end-effector, one revolute (R) joint connects the distal and proximal links of each leg, and another revolute $(R)$ joint connects the proximal link of each leg to the body. The three revolute joints between the proximal links and the body are actuated by the servo motors. In each leg, the axes of the two revolute joints are parallel, so that it constitutes a 2-DoF planar articulated mechanism, constraining the motion of the center of each spherical joint on a plane fixed w.r.t. the body. The mobile end-effector has therefore 3-DoF w.r.t. the body. We used three PWMcontrolled HS-5035HD servomotors (HiTech, Republic of Korea). The wearable device weights $25 \mathrm{~g}$ for $35 \times 50 \times 48 \mathrm{~mm}$ dimensions.

Control. Let $\boldsymbol{\xi}=\left[\begin{array}{llllll}p_{x} & p_{y} & p_{z} & \psi & \theta & \phi\end{array}\right]^{\mathrm{T}}$ be the Cartesian pose of the platform, being $\xi_{0}$ the reference pose in the non-contact condition. When in contact with the finger, any (small) displacement $\Delta \xi=\xi-\xi_{0}$ of the platform leads to a contact stress distribution on the finger pad. The resultant force $\mathbf{f}_{p}$ and moment $\mathbf{m}_{p}$ of the normal and tangential stress distributions, arising at the contact patch, balance the external wrench $-\mathbf{w}_{p}$. Fingertip deformation and applied wrench can be related by an impedance model, which is typically nonlinear and depends on the fingertip specific 
characteristics (e.g., geometric parameters, subject's age) as well as on the actual device configuration q. In this work, we assume a simplified fingertip impedance model, namely a linear (constant) relationship between the resultant wrench and the platform displacement around the reference pose of the device. In other terms, we consider the platform displacement $\Delta \xi$ proportional to the wrench $\mathbf{w}_{p}$,

$$
\mathbf{w}_{p}=K \Delta \xi
$$

where $K \in \mathbb{R}^{6 \times 6}$ is the fingertip stiffness matrix, as defined in [32], [33]. From a desired wrench $\mathbf{w}_{p, d}$, we can therefore evaluate the corresponding desired platform pose $\xi_{d}=\xi_{0}+K^{-1} \mathbf{w}_{p, d}$ to be actuated through the servo motors. Since the device has $3 \mathrm{DoF}$, only three of the six parameters describing the platform pose can be controlled. For our application, we seek to control the position along the $z$ direction, $p_{z, d}$, and the roll $\psi_{d}$ and pitch $\theta_{d}$ angles. As shown in Fig. 1c, the desired wrench $\mathbf{w}_{p, d}$ is compared with the computed one $\hat{\mathbf{w}}_{p}$. Inverting eq. (1), the error in the wrench space $\Delta \mathbf{w}_{p}$ is transformed into a desired displacement on the platform pose $\Delta \xi_{d}$, which is then transformed into a desired displacement of the device configuration $\Delta \mathbf{q}_{d}$ through an inverse kinematics procedure. A PID controller is used to evaluate the torque $\tau$ to be applied by the device actuators. The device dynamics will therefore depend on the applied torque $\tau$ and on the actual wrench $\mathbf{w}_{p}$ due to the interaction with the fingertip. Finally, incremental encoders on the servo motors measure their actual rotation $\Delta \mathbf{q}$, and then, through a direct kinematic procedure, we evaluate the actual platform displacement $\Delta \xi$. More details on the control of this device can be found in [30].

\section{2 hRing finger device}

Description. The hRing wearable finger device has been preliminarily presented by Pacchierotti et al. [31], and it is shown in Fig. 2. It is composed of a static part, that houses two servo motors and two pulleys, and a fabric belt, that applies the requested stimuli to the finger. A strap band is used to secure the device on the finger proximal phalanx. We used two PWM-controlled HS-40 servomotors (HiTech, Republic of Korea). The device weights $38 \mathrm{~g}$ for $30 \times 43 \times 25 \mathrm{~mm}$ dimensions.

Control. The working principle of the device is depicted in Fig. 2b. Similarly to the principle proposed by Minamizawa et al. [34], when the two motors rotate in opposite directions, the belt is pulled up, providing a force normal to the finger (left side of Fig. 2b). On the other hand, when motors spin in the same direction, the belt applies a shear force to the finger (right side of Fig. 2b). Of course, these two movements can be combined together to provide at the same time shear and normal stimuli.

As for the 3-RRS fingertip device, the servomotors are position controlled, which means that it is only possible to command a desired angle. The relationship between the commanded angle and belt displacement is

$$
\Delta d=r \Delta \theta_{c},
$$

where $r=5 \mathrm{~mm}$ is the radius of the servo motor pulley, $\Delta d$ the belt displacement, and $\Delta \theta_{c}$ the commanded angle expressed in radians. To relate the belt displacement to the desired wrench to apply on the finger proximal phalanx $\mathbf{w}_{\mathbf{p}, \mathbf{d}}$, we assume again

$$
\mathbf{w}_{p, d}=K \Delta d
$$

where $K \in \mathbb{R}^{6 \times 6}$ is the finger phalanx stiffness matrix [32], [33] and $\Delta d$ is the displacement of the belt since it first made contact with the fingertip. Despite the simplicity of actuation, it has been demonstrated that the vertical and shearing forces generated by the deformation of the fingerpads can reproduce reliable weight sensations even when proprioceptive sensations on the wrist and the arm are absent [35].

\section{EXPERIMENTAL EVALUATION}

In order to understand and analyze the role, potential, and effectiveness of employing wearable haptic interfaces in AR scenarios, we carried out three experiments.

\subsection{Experiment \#1: writing on a whiteboard}

In the first experiment, subjects are asked to write on a virtual white board while holding a real chalk. Here we aim at evaluating how wearable haptics can improve the performance of the writing task on top of standard sensory substitution techniques.

\subsubsection{Setup}

The experimental setup is composed of a video camera, a visual marker, a screen, a chalk, and a virtual whiteboard, as shown in Fig. 3a. Subjects are requested to hold the chalk as if they are going to write on a real board. The visual marker, fixed on the top of the chalk, enables the system to create the virtual whiteboard in front of the subject. The whiteboard has already four letters written on it, "CIAO", which subjects are required to trace with the chalk.

\subsubsection{Subjects}

Fourteen participants (10 males, 4 females, age range 21 - 36) took part to the experiment, all of whom were right-handed. Seven of them had previous experience with haptic interfaces. None of the participants reported any deficiencies in their perception abilities and they were all naïve as to the purpose of the study.

\subsubsection{Methods}

The task consisted in tracing the word "CIAO" on the virtual whiteboard as precisely as possible and applying as little force as possible to the board. The task started when the subject touched the whiteboard for the very first time and finished when the subject completed the tracing. As the chalk touches the virtual whiteboard, a suitable amount of force $\mathbf{f}_{\mathbf{p}}$ is provided to the user, according to the feedback modality considered. This interaction force, applied by the chalk on the virtual board, is evaluated according to the god-object model [36]. We modeled the chalk-whiteboard interaction with a spring of stiffness $500 \mathrm{~N} / \mathrm{m}$, e.g., a penetration of $5 \mathrm{~mm}$ inside the virtual whiteboard produced a force of $2.5 \mathrm{~N}$. A video of this experiment is available as supplemental material. 


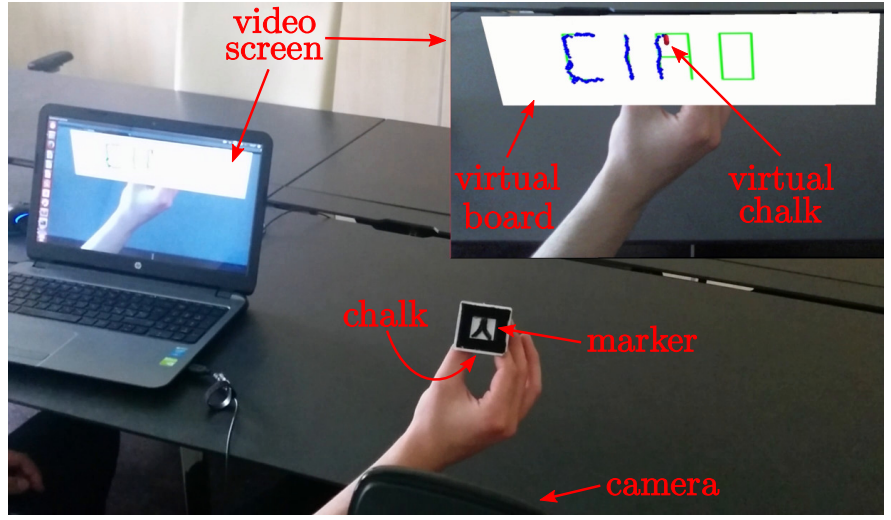

(a) Experimental setup.

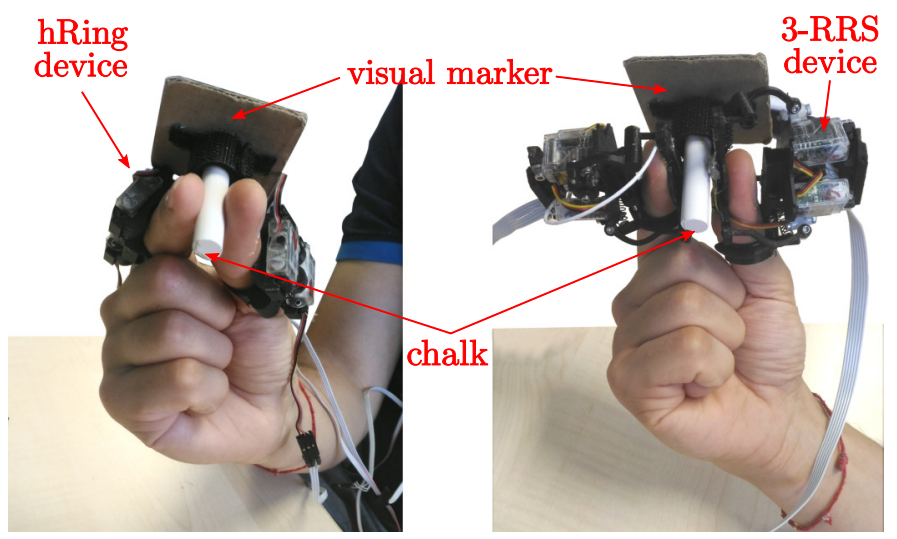

(b) Subject holding the chalk while wearing the hRing and the 3-RRS device.

Fig. 3. Experiment \#1: writing on a whiteboard. Experimental setup Subjects are required to hold the chlak supporting the marker and trace the word on the virtual whiteboard.

Each participant made fifteen trials of the writing task, with three randomized repetitions for each feedback condition proposed:

(K) kinesthetic feedback provided by the Omega.6 haptic interface,

$(\mathrm{CR}+\mathrm{V})$ cutaneous feedback provided by the hRing cutaneous device and sensory substitution via visual feedback,

$(\mathrm{CF}+\mathrm{V})$ cutaneous feedback provided by the 3-RRS cutaneous device and sensory substitution via visual feedback,

(V) sensory substitution via visual feedback,

(N) no force feedback.

In condition $\mathrm{K}$, the marker is attached on the pen-shaped end-effector of the Omega.6 interface. Subjects are asked to hold the end-effector of the Omega as they would hold a chalk (no chalk is present in this condition). As the user touches the virtual whiteboard, the Omega provides kinesthetic feedback perpendicular to the board.

In condition $\mathrm{CR}+\mathrm{V}$, subjects are required to hold the chalk housing the marker and wear two hRing devices, on the thumb and index fingers (see Fig. 3b). As the user touches the virtual whiteboard, the hRing devices provide skin stretch feedback perpendicular to the board, as described in Sec. 2.2. Also the color of the mark changes according to the amount of force exerted on the board, from blue to red. A blue mark indicates a light touch, while a red mark indicates a heavy touch, i.e.,

$$
\begin{aligned}
& \left\{\begin{array}{l}
R=51\left\|\mathbf{f}_{\mathbf{p}}\right\| \\
G=0 \\
B=255-51\left\|\mathbf{f}_{\mathbf{p}}\right\|
\end{array} \quad \text { if }\left\|\mathbf{f}_{\mathbf{p}}\right\| \leq 5 \mathrm{~N},\right. \\
& \left\{\begin{array}{l}
R=255 \\
\begin{array}{l}
G=0 \\
B
\end{array}=0
\end{array}\right.
\end{aligned}
$$

A similar use of visual cues to substitute force feedback was adopted also in [37].

In condition $\mathrm{CF}+\mathrm{V}$, subjects are required to hold the chalk housing the marker and wear two 3-RRS devices, on the thumb and index fingers (see Fig. 3b). As the user touches the virtual whiteboard, the 3-RRS fingertip devices provide contact deformation feedback perpendicular to the board, as described in Sec. 2.1. As before, also the color of the mark changes according to the amount of force exerted on the board, from blue to red (see Eq. (4)).

In condition $\mathrm{V}$, subjects are required to hold the chalk housing the marker, but no devices are worn on the fingers. As in conditions $\mathrm{CR}+\mathrm{V}$ and $\mathrm{CF}+\mathrm{V}$, the color of the mark changes according to the amount of force exerted on the board.

In condition $\mathrm{N}$, subjects are required to hold the chalk housing the marker. No devices are worn on the fingers, and no information about the forces exerted on the virtual board is provided to the user. The color of the mark is always blue, regardless of the value of $\left\|\mathbf{f}_{\mathbf{p}}\right\|$.

Our hypothesis is that providing information about the interaction forces between the (real) chalk and the (virtual) white board reduces the force applied by the user on the augmented environment.

\subsubsection{Results}

As a measure of performance, we evaluated (1) the error in tracing the word "CIAO" on the virtual whiteboard, (2) the force exerted by the chalk on the board, (3) the total distance traveled by the chalk, and (4) the completion time. To compare the different metrics, we ran one-way repeatedmeasures ANOVAs. The feedback condition was treated as the within-subject factor.

Figure 4a shows the error in tracing the word "CIAO" on the virtual whiteboard, calculated as the mean difference between the trace written by the subject and the original word. Data was transformed using the squareroot transformation. Transformed data passed the ShapiroWilk normality test and the Mauchly's Test of Sphericity. The one-way repeated-measure ANOVA revealed statistically significant difference between the feedback conditions $\left(F_{4,52}=26.981, p<0.001, a=0.05\right)$. Post hoc analysis with Bonferroni adjustments revealed a statistically significant difference between conditions $\mathrm{CR}+\mathrm{V}$ vs. $\mathrm{K}(p=0.020), \mathrm{CR}+\mathrm{V}$ vs. $\mathrm{V}(p=0.011), \mathrm{CR}+\mathrm{V}$ vs. $\mathrm{N}(p=0.006), \mathrm{K}$ vs. $\mathrm{CF}+\mathrm{V}$ $(p=0.036), \mathrm{K}$ vs. $\mathrm{V}(p<0.001), \mathrm{K}$ vs. $\mathrm{N}(p<0.001), \mathrm{CF}+\mathrm{V}$ vs. $\mathrm{V}(p<0.001), \mathrm{CF}+\mathrm{V}$ vs. $\mathrm{N}(p=0.001)$. The Bonferroni correction is used to reduce the chances of obtaining falsepositive results when multiple pair-wise tests are performed on a single set of data.

Figure $4 \mathrm{~b}$ shows the force exerted by the users on the virtual board, calculated as the root mean square of $\left\|\mathbf{f}_{\mathbf{p}}\right\|$ (see 


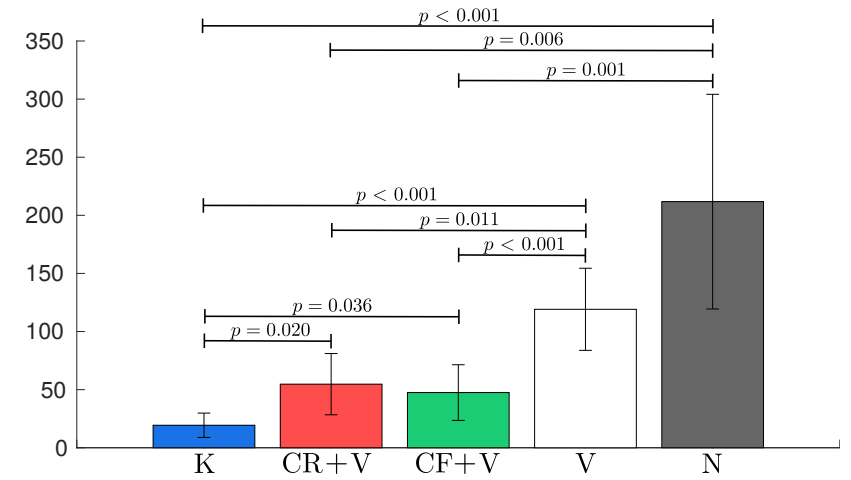

(a) Mean error ( $\mathrm{mm})$.

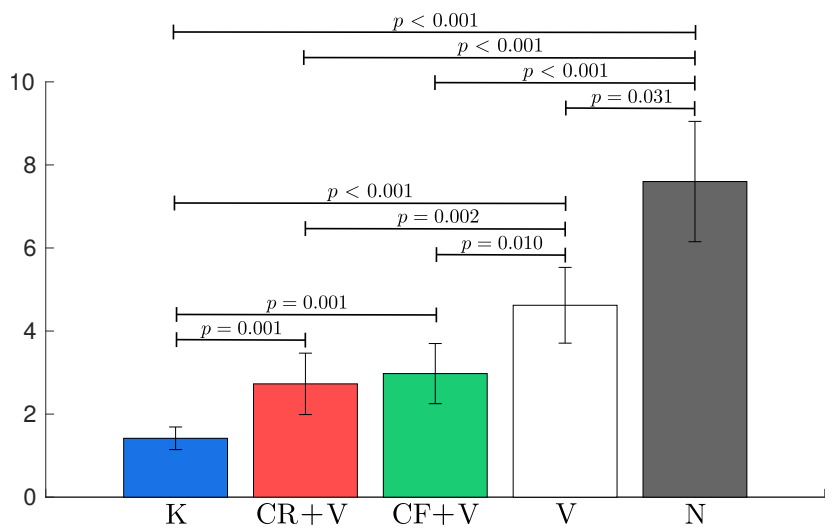

(b) Force $(\mathrm{N})$.

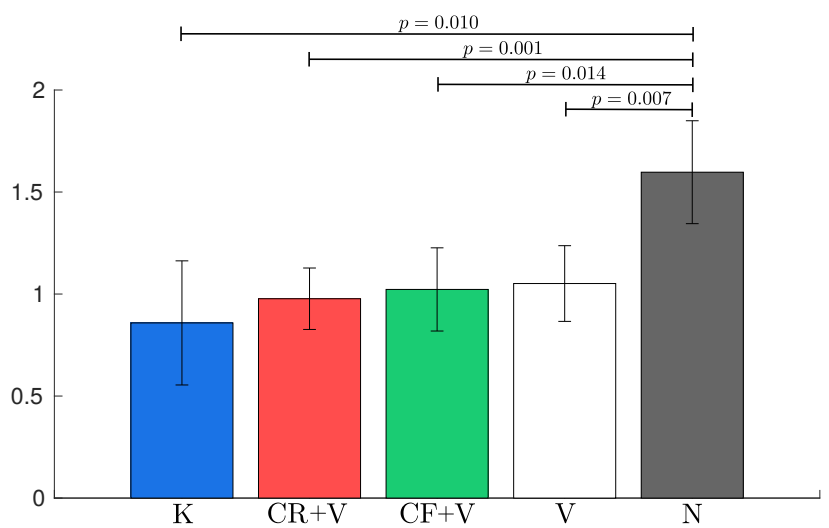

(c) Overall distance (m).

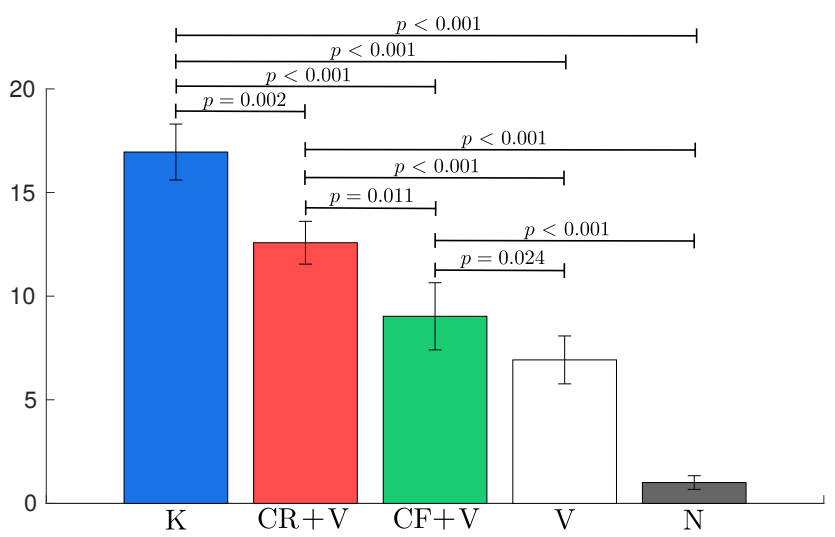

(d) Perceived effectiveness.

Fig. 4. Experiment \#1. Mean and standard deviation of (a) the mean error, (b) the interaction force, (c) total distance traveled by the chalk, and (d) the perceived effectivess of the five feedback conditions are plotted.
Sec. 3.1.3). The data passed the Shapiro-Wilk normality test. Mauchly's Test of Sphericity indicated that the assumption of sphericity had been violated $\left(\chi^{2}(9)=29.859, p=0.001\right)$ The one-way repeated-measure ANOVA with a GreenhouseGeisser correction revealed statistically significant difference between the feedback conditions $\left(F_{1.794,23.326}=37.899, p<\right.$ $0.001, a=0.05)$. Post hoc analysis with Bonferroni adjustments revealed a statistically significant difference between conditions $\mathrm{K}$ vs. $\mathrm{CR}+\mathrm{V}(p=0.001), \mathrm{K}$ vs. $\mathrm{CF}+\mathrm{V}(p=0.001)$, $\mathrm{K}$ vs. $\mathrm{V}(p<0.001), \mathrm{K}$ vs. $\mathrm{N}(p<0.001), \mathrm{CR}+\mathrm{V}$ vs. $\mathrm{V}$ $(p=0.002), \mathrm{CR}+\mathrm{V}$ vs. $\mathrm{N}(p<0.001), \mathrm{CF}+\mathrm{V}$ vs. V $(p=0.010)$, $\mathrm{CF}+\mathrm{V}$ vs. $\mathrm{N}(p<0.001), \mathrm{V}$ vs. $\mathrm{N}(p=0.031)$.

After this variance analysis, we also tested whether the force applied has any relationship with the measured error in tracing the word. Our hypothesis is that being provided with information about the force applied on the board, make subjects concentrate more on the task, achieving also smaller tracing errors. A Pearson's product-moment correlation was run to assess the relationship between the root mean square of $\left\|\mathbf{f}_{\mathbf{p}}\right\|$ and the tracing error. Preliminary analyses showed the relationship to be linear with variable normally distributed, as assessed by Shapiro-Wilk test, and that there were no outliers. There was a strong positive correlation between the two metrics $(r(70)=0.780, p<0.001)$, confirming our hypothesis.

Figure $4 \mathrm{c}$ shows the total distance traveled by the chalk during the task. Data was transformed using the log transformation. Transformed data passed the ShapiroWilk normality test. Mauchly's Test of Sphericity indicated that the assumption of sphericity had been violated $\left(\chi^{2}(9)=32.154, p<0.001\right)$. The one-way repeated-measure ANOVA with a Greenhouse-Geisser correction revealed statistically significant difference between the feedback conditions $\left(F_{2.182,28.364}=10.460, p<0.001, a=0.05\right)$. Post hoc analysis with Bonferroni adjustments revealed a statistically significant difference between conditions $\mathrm{CR}+\mathrm{V}$ vs. $\mathrm{N}(p=0.001), \mathrm{K}$ vs. $\mathrm{N}(p=0.010), \mathrm{CF}+\mathrm{V}$ vs. $\mathrm{N}$ $(p=0.014), \mathrm{V}$ vs. $\mathrm{N}(p=0.007)$.

We also measured the task completion time (not shown in the figures). The task started when the subject touched the whiteboard for the very first time and finished when the subject completed the tracing. The registered mean time was 110 s. All the data passed the Shapiro-Wilk normality test and the Mauchly's Test of Sphericity. The one-way repeated-measure ANOVA revealed no statistically significant difference between the feedback conditions $\left(F_{4,52}=1.951, p=0.116, a=0.05\right)$.

In addition to the quantitative evaluation reported above, we also measured users' experience. At the end of the experiment, subjects were asked to rate, on a slider going from 0 to 20 , the effectiveness of each feedback condition in completing the given task. Figure $4 \mathrm{~d}$ shows the perceived effectiveness for the five feedback conditions. Data passed the Shapiro-Wilk normality test and the Mauchly's Test of Sphericity. A one-way repeated-measure ANOVA revealed statistically significant difference between the feedback conditions $\left(F_{4,52}=116.670, p<0.001, a=0.05\right)$. Post hoc analysis with Bonferroni adjustments revealed a statistically significant difference between conditions $\mathrm{CR}+\mathrm{V}$ vs. $\mathrm{K}(p=0.002), \mathrm{CR}+\mathrm{V}$ vs. $\mathrm{CF}+\mathrm{V}(p=0.011), \mathrm{CR}+\mathrm{V}$ vs. $\mathrm{V}$ $(p<0.001), \mathrm{CR}+\mathrm{V}$ vs. $\mathrm{N}(p<0.001), \mathrm{K}$ vs. $\mathrm{CF}+\mathrm{V}(p<0.001)$, 


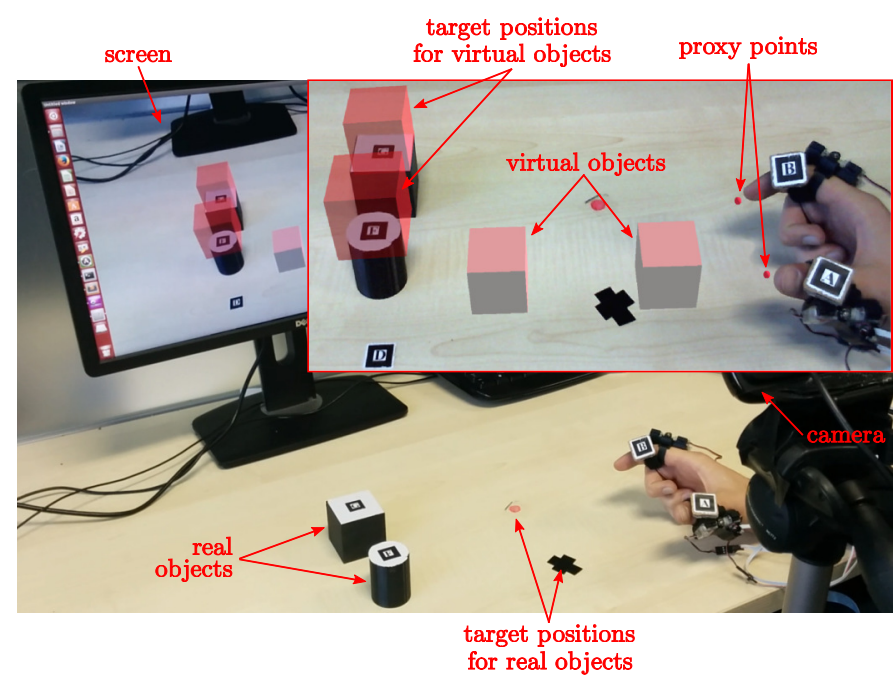

Fig. 5. Experiment \#2: pick and place. Experimental setup. Subjects need to pick up the four objects, one by one, and place them into their corresponding target positions.

$\mathrm{K}$ vs. $\mathrm{V}(p<0.001), \mathrm{K}$ vs. $\mathrm{N}(p<0.001), \mathrm{CF}+\mathrm{V}$ vs. $\mathrm{V}$ $(p=0.024), \mathrm{CF}+\mathrm{V}$ vs. $\mathrm{N}(p<0.001)$.

\subsection{Experiment \#2: pick and place}

In this second experiment, subjects are asked to pick and place virtual and real objects. Differently from before, in this experiment we have separated the conditions providing cutaneous and sensory substitution feedbacks, in order to better understand the role of cutaneous cues. Moreover, as we seek for lightweight, compact, and wearable solutions, we did not include conditions providing force feedback through grounded interfaces.

\subsubsection{Setup}

The experimental setup is composed of a video camera, five visual markers, a screen, two real objects, and two virtual objects, as shown in Fig. 5. Two markers are worn by the subjects on the thumb and index middle phalanges, two markers are placed on the real objects, and one marker is attached on the table supporting the environment. From each marker worn by the subjects, the AR system creates a proxy point, roughly positioned at the fingertip of the corresponding finger. All the interactions between the finger and the virtual objects are mediated by these points. Target positions for the real objects are marked on the table, while target positions for the virtual objects are represented as transparent volumes. When at least half of the virtual object is inserted into the corresponding volume, the latter turns green to indicate a correct positioning.

\subsubsection{Subjects}

14 participants (9 males, 3 females, age range $25-35$ ) took part to the experiment, all of whom were right-handed. Eight of them had previous experience with haptic interfaces. None of the participants reported any deficiencies in their perception abilities and they were all naïve as to the purpose of the study.

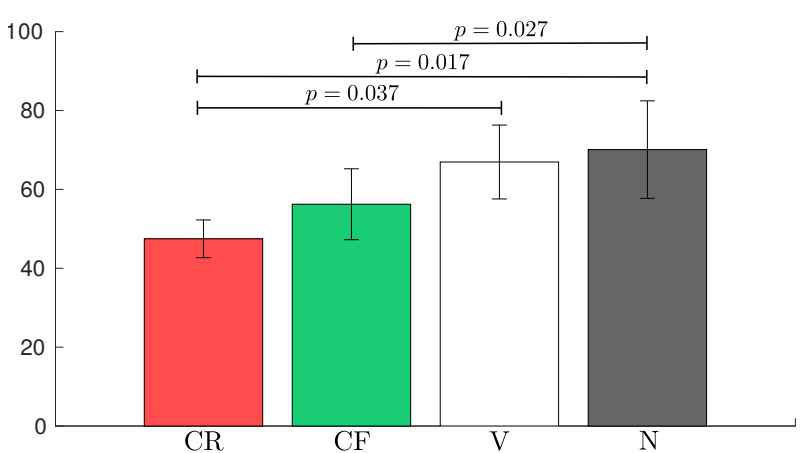

(a) Completion time (s).

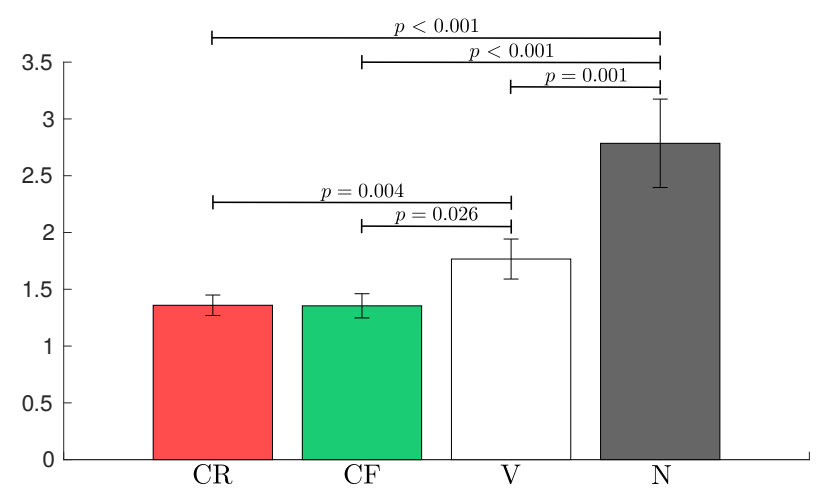

(b) Force (N).

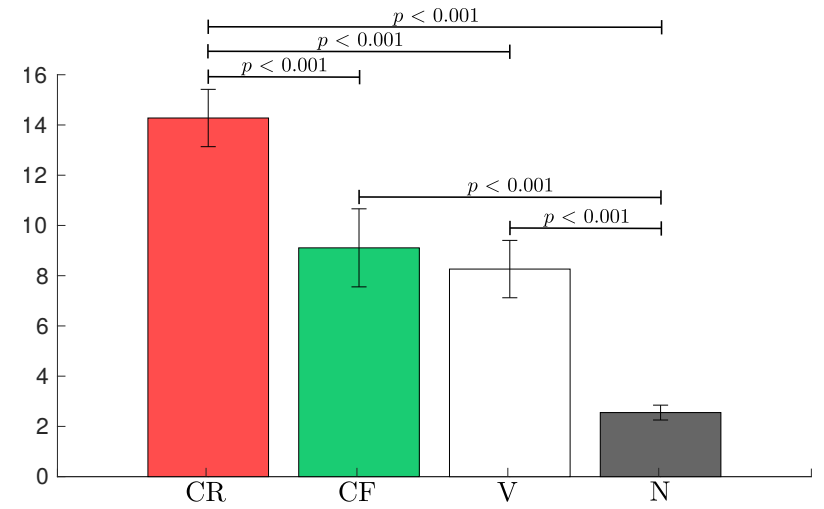

(c) Perceived effectiveness.

Fig. 6. Experiment \#2. Mean and standard deviation of (a) the completion time, (b) force exerted by the users on the virtual objects, and (c) perceived effectivess of the four feedback conditions are plotted.

\subsubsection{Methods}

The task consisted of picking up the four objects, one by one, and placing them into their corresponding target positions. The task is completed when all the four objects are placed at the corresponding target locations. As the index and thumb fingertips touch the virtual objects, a suitable amount of force $f_{p}$ is provided to the user, according to the feedback modality considered. This interaction force is evaluated according to the god-object model [36]. As for the first experiment, we modeled the object-fingertip interaction with a spring of stiffness $500 \mathrm{~N} / \mathrm{m}$. A video of this experiment is available as supplemental material.

Each participant made twelve trials of the pick and place task, with three randomized repetitions for each feedback condition proposed: 
(CR) cutaneous feedback provided by the hRing cutaneous device,

(CF) cutaneous feedback provided by the 3-RRS cutaneous device,

(V) sensory substitution via visual feedback,

(N) no force feedback.

In condition $\mathrm{CR}$, subjects are required to wear two visual markers and two hRing devices, on the thumb and index fingers (as in Fig. 5). As the user touches a virtual object, the hRing devices provide skin stretch feedback perpendicular to the surface being touched, as described in Sec. 2.2.

In condition $C F$, subjects are required to wear two visual markers and two 3-RRS devices, on the thumb and index fingers. As the user touches a virtual object, the 3-RRS devices provide contact deformation feedback perpendicular to the surface being touched, as described in Sec. 2.1.

In condition $\mathrm{V}$, subjects are required to wear two visual markers but no cutaneous devices. As the user touches a virtual object, the color of the object changes according to the amount of force exerted on it, from red to black. A red shade of the object indicates a light grasp, while a black shade indicates a strong grasp, i.e.,

$$
\begin{aligned}
& \left\{\begin{array}{l}
R=255-51\left\|\mathbf{f}_{\mathbf{p}}\right\| \\
G=0 \\
B=0
\end{array}\right. \\
& \begin{cases}R=0 \\
G=0 \\
B=0\end{cases}
\end{aligned}
$$

In condition $\mathrm{N}$, subjects are required to wear two visual markers. No devices are worn on the fingers, and no information about the interaction forces are provided to the user.

Our hypothesis is that providing information about the grasping forces reduces the force applied by the user on the augmented environment. Moreover, we also expect the hRing to be preferred by the users, as it enables a direct interaction with the real objects.

\subsubsection{Results}

As a measure of performance, we evaluated (1) the completion time, (2) the force applied by the users to the virtual objects, and (3) the total distance traveled by the fingers. To compare the different metrics, we ran one-way repeatedmeasures ANOVAs. The feedback condition was treated as the within-subject factor.

Figure $6 \mathrm{a}$ shows the task completion time. The data passed the Shapiro-Wilk normality test and the Mauchly's Test of Sphericity. The one-way repeated-measure ANOVA revealed statistically significant difference between the feedback conditions $\left(F_{3,39}=7.828, p<0.001, a=0.05\right)$. Post hoc analysis with Bonferroni adjustments revealed a statistically significant difference between conditions CR vs. $\mathrm{V}(p=0.037), \mathrm{CR}$ vs. $\mathrm{N}(p=0.017)$, and CF vs. $\mathrm{N}$ $(p=0.027)$.

Figure $6 \mathrm{~b}$ shows the force exerted by the users on the virtual objects, calculated as the root mean square of $\left\|\mathbf{f}_{\mathbf{p}}\right\|$ (see Sec. 3.2.3). The data passed the Shapiro-Wilk normality test. Mauchly's Test of Sphericity indicated that the assumption of sphericity had been violated $\left(\chi^{2}(5)=26.666, p<0.001\right)$.
The one-way repeated-measure ANOVA with a GreenhouseGeisser correction revealed statistically significant difference between the feedback conditions $\left(F_{1.622,21.088}=42.349, p<\right.$ $0.001, a=0.05$ ). Post hoc analysis with Bonferroni adjustments revealed a statistically significant difference between conditions $\mathrm{CR}$ vs. $\mathrm{V}(p=0.004), \mathrm{CR}$ vs. $\mathrm{N}(p<0.001), \mathrm{CF}$ vs. $\mathrm{V}(p=0.026), \mathrm{CF}$ vs. $\mathrm{N}(p<0.001), \mathrm{V}$ vs. $\mathrm{N}(p=0.001)$.

We also measured the total distance traveled by the fingers (not shown in the figures). The data passed the Shapiro-Wilk normality test and the Mauchly's Test of Sphericity. The one-way repeated-measure ANOVA revealed no statistically significant difference between the feedback conditions $\left(F_{3,39}=1.789, p=0.165, a=0.05\right)$.

As for the previous experiment, in addition to the quantitative evaluation reported above, we also measured users' experience. At the end of the experiment, subjects were asked to rate, on a slider going from 0 to 20 , the effectiveness of each feedback condition in completing the given task. Figure $6 \mathrm{c}$ shows the perceived effectiveness for the four feedback conditions. The data passed the Shapiro-Wilk normality test. Mauchly's Test of Sphericity indicated that the assumption of sphericity had been violated $\left(\chi^{2}(5)=14.817, p=0.011\right)$. The one-way repeated-measure ANOVA with a Greenhouse-Geisser correction revealed statistically significant difference between the feedback conditions $\left(F_{2.037,26.480}=86.945, p<0.001, a=0.05\right)$. Post hoc analysis with Bonferroni adjustments revealed a statistically significant difference between conditions $C R$ vs. CF $(p<0.001)$, CR vs. V $(p<0.001)$, CR vs. $\mathrm{N}(p<0.001)$, CF vs. $\mathrm{N}(p<0.001)$, V vs. $\mathrm{N}(p<0.001)$.

\subsection{Experiment \#3: ball and plate}

In this third experiment, subjects are asked to balance a virtual sphere on a real cardboard. This time we made use of a VR headset to improve the illusion of presence in the augmented reality environment.

\subsubsection{Setup}

The experimental setup is composed of a video camera mounted on an Oculus Rift Head-Mounted Display (HMD), a flat cardboard, and a visual marker, as shown in Fig. 7. The AR system creates four walls at the edges of the cardboard, a virtual sphere inside these walls, and a target volume for the virtual sphere. With respect to the other experiments, subjects do not see the augmented environment through a LCD screen, but they use the Oculus Rift HDM, which is able to provide a compelling and subjective view of the augmented scenario.

\subsubsection{Subjects}

10 participants ( 9 males, 1 females, age range $25-35$ ) took part to the experiment, all of whom were right-handed. Seven of them had previous experience with haptic interfaces. None of the participants reported any deficiencies in their perception abilities and they were all naïve as to the purpose of the study.

\subsubsection{Methods}

The task consisted in moving a virtual sphere on a plane cardboard toward multiple target locations. Subjects are 


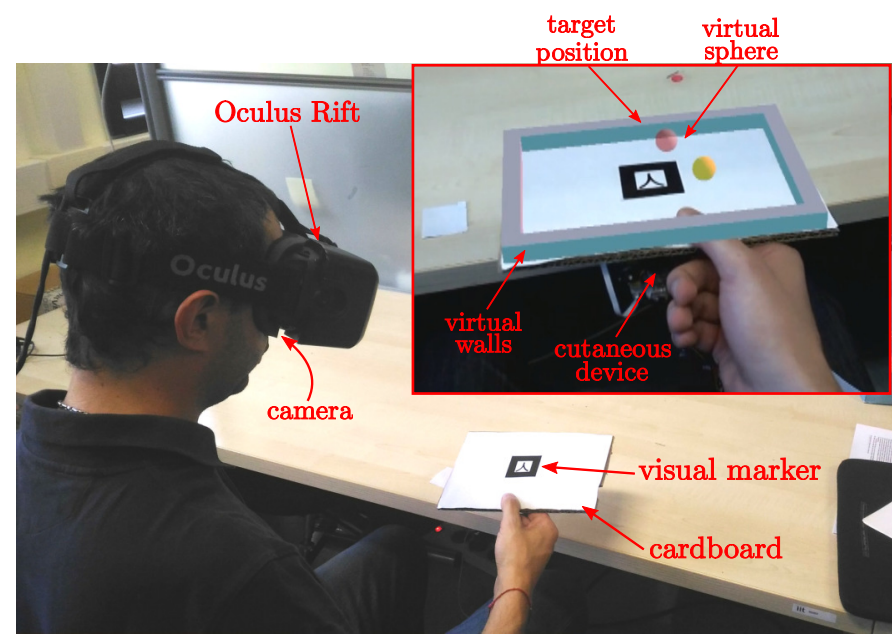

Fig. 7. Experiment \#3: ball and plate. Experimental setup. Subjects hold the cardboard with their thumb and index fingers, wearing one device on the index, and incline the cardboard to move the sphere toward the indicated target location.

required to hold the cardboard with their thumb and index fingers, and incline the cardboard to move the sphere toward the indicated target location (see Fig. 7). Once the sphere has reached the current target location, the AR system randomly spawns another target location for the sphere to reach, and so on. We registered how many spheres the user can place in $45 \mathrm{~s}$.

A video of this experiment is available as supplemental material.

Each participant made nine trials of the ball and plate task, with three randomized repetitions for each feedback condition proposed:

$(\mathrm{CR}+\mathrm{Vb})$ cutaneous feedback provided by the hRing cutaneous device and a vibrotactile motor,

$(\mathrm{CF}+\mathrm{Vb})$ cutaneous feedback provided by the 3-RRS cutaneous device and a vibrotactile motor,

(N) no force feedback.

In condition $\mathrm{CR}+\mathrm{Vb}$, subjects are required to hold the cardboard and wear one hRing device on the index finger. The index finger is used to sustain the cardboard and the hRing conveys the additional weight of the virtual sphere. Moreover, a vibrotactile motor attached on the back of the cardboard conveys 100-ms-long vibration bursts every time the sphere touches the side walls.

In condition $\mathrm{CF}+\mathrm{Vb}$, subjects are required to hold the cardboard and wear one 3-RRS device on the index fingertip. The index finger is used to sustain the cardboard and the 3-RRS device conveys the additional weight of the virtual sphere. As before, a vibrotactile motor conveys vibration bursts every time the sphere touches the side walls.

In condition $\mathrm{N}$, subjects wear no devices and no information about the interaction of the sphere with the cardboard is provided.

Our hypothesis is that the two degrees of freedom of the hRing device will not be enough to effectively render the inclination of the platform on the finger pulp. For this reason, we expect the 3-RRS device to perform better and to be preferred by the users.

\subsubsection{Results}

As a measure of performance, we evaluated the number of spheres placed in $45 \mathrm{~s}$.

Figure $8 \mathrm{a}$ shows the total number of spheres placed. The data passed the Shapiro-Wilk normality test. Mauchly's Test of Sphericity indicated that the assumption of sphericity had been violated $\left(\chi^{2}(2)=7.349, p=0.025\right)$. The oneway repeated-measure ANOVA with a Greenhouse-Geisser correction revealed statistically significant difference between the feedback conditions $\left(F_{1.249,11.243}=20.102, p=\right.$ $0.001, a=0.05$ ). Post hoc analysis with Bonferroni adjustments revealed a statistically significant difference between conditions CR vs. CF ( $p=0.008), \mathrm{CR}$ vs. $\mathrm{N}(p=0.018), \mathrm{CF}$ vs. $\mathrm{N}(p=0.001)$.

As for the previous experiment, in addition to the quantitative evaluation reported above, we also measured users' experience. At the end of the experiment, subjects were asked to rate, on a slider going from 0 to 20 , the effectiveness of each feedback condition in completing the given task. Figure $8 \mathrm{~b}$ shows the perceived effectiveness for the three feedback conditions. The data passed the ShapiroWilk normality test and the Mauchly's Test of Sphericity. The one-way repeated-measure ANOVA revealed statistically significant difference between the feedback conditions $\left(F_{2,18}=24.091, p<0.001, a=0.05\right)$. Post hoc analysis with Bonferroni adjustments revealed a statistically significant difference between conditions CR vs. CF $(p=0.002)$ and CF vs. $\mathrm{N}(p=0.003)$.

\section{Discussion}

We tested the role of two finger wearable haptic devices in three augmented reality scenarios. The first device, referred to as 3-RRS device, is able to provide 3-DoF contact stimuli at the fingertip. The second device, referred to as hRing, is able to provide normal and skin stretch stimuli to the user's proximal finger phalanx.

In the first experiment, subjects are asked to write on a virtual board using a real chalk. The haptic devices provide the interaction forces between the chalk and the board. Results presented in Sec. 3.1.4 show that kinesthetic feedback provided by the grounded interface (condition K) greatly outperforms all the other feedback modalities. However, as we have discussed in Sec. 1, AR applications often seek for small and lightweight devices, which can be easily worn and carried around. Commercially-available grounded interfaces, such as the Omega.6 we employed, do not fall into this category. Among the other feedback conditions, cutaneous stimuli provided either through the 3-RRS device $(\mathrm{CF}+\mathrm{V})$ or the $h$ Ring device $(\mathrm{CR}+\mathrm{V})$ performed the best. No significant difference between the two cutaneous conditions was found except for the user's subjective evaluation, where subjects preferred the hRing device. The main reason for this choice was the fact that the interaction force $\mathbf{f}_{p}$ between the chalk and the virtual whiteboard mainly produced stimuli tangential to the fingertip skin (see Fig. 3b), which are best rendered by the hRing device. In fact, the rigid platform of the 3-RRS device is not able to provide purely tangential/stretch stimuli to the fingertip. A detailed discussion on the maximum tangential force the 3-RRS device can apply can be found in [38]. Surprisingly, this lack of actuation capabilities of the 


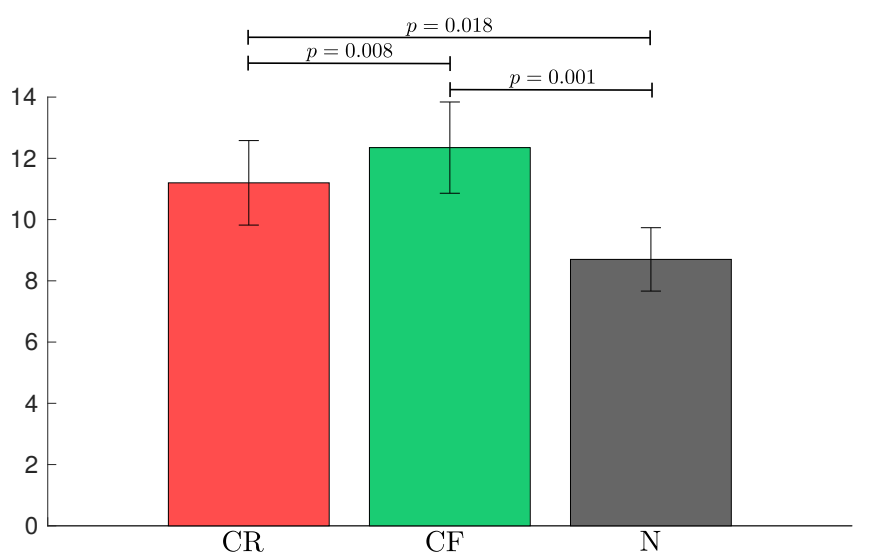

(a) Number of spheres placed.

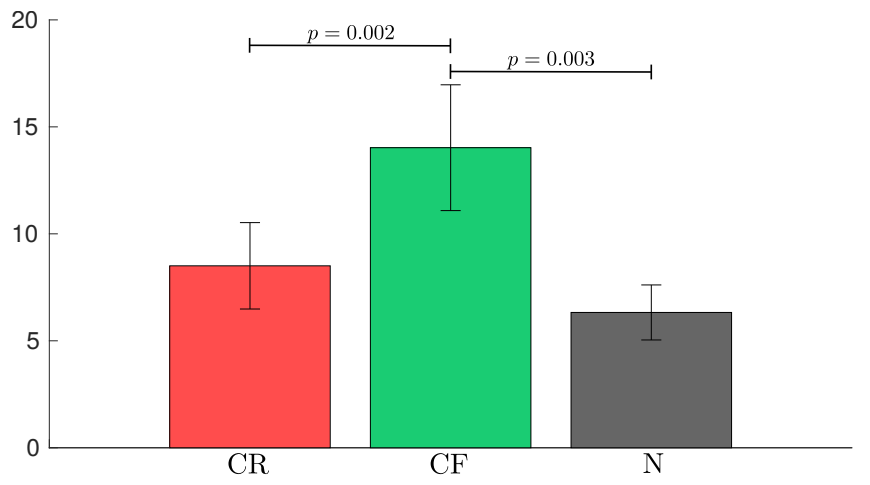

(b) Perceived effectiveness.

Fig. 8. Experiment \#3. Mean and standard deviation of (a) the number of spheres placed in $45 \mathrm{~s}$ and (b) the perceived effectivess of the three feedback conditions are plotted.

3-RRS device did not lead to a significant degradation of the other metrics. Finally, also simply changing the color of the chalk mark according to $\mathbf{f}_{p}$ helped the user in applying less force (see V vs. N). It is also interesting to notice that providing information about the force applied on the whiteboard directly affected the tracing error (see Fig. 4a vs. Fig. $4 \mathrm{~b}$ and correlation test). We believe that being provided with this information made the subjects concentrate more on the task, resulting in a smaller force and tracing error.

In the second experiment, subjects are asked to pick and place virtual and real objects. The haptic devices provide information about the weight of the virtual objects being picked up. For the reasons discussed above, here we did not include kinesthetic feedback provided by a grounded haptic interface. Moreover, in order to better understand the role of cutaneous stimuli, we decoupled conditions providing cutaneous feedback (CR and $\mathrm{CF}$ ) from those providing sensory substitution cues via visual feedback (V). Similarly as before, also here the cutaneous stimuli provided either through the 3-RRS device (CF) or the hRing device (CR) performed the best. No significant difference between the two cutaneous conditions was found except for the user's subjective evaluation, where subjects preferred again the hRing device. The main reason for this choice was the fact that the interaction force due to the weight of virtual objects mainly produced stimuli tangential to the fingertip skin which are best rendered by the hRing device. However, as in the first experiment, the lack of pure shear forces in the 3-RRS device did not lead to a significant degradation of the other metrics. This behavior may suggest that also a slanted surface can provide a good illusion of weight. A similar result had also been observed in [38], where the authors used a 3-DoF rigid surface at the fingertip to render the weight of remote objects. Finally, 11 participants out of the 14 we enrolled complained that the 3-RRS device severely limited the interaction with real objects, since the rigid platform prevented the fingertip from touching the environment. Conversely, the hRing device left the subjects' fingertips free. Indeed, this is a relevant design issue for wearable haptic devices in AR applications. While it is important to provide haptic stimuli exactly where they are expected to be (i.e., the fingertip in our case) [39], placing a rigid end-effector at the contact point may interfere with the interaction with real objects (see right side of Fig. 3b). A promising solution is to move the end-effector away from the contact location, as in our hRing device. Doing so, users can still receive haptic stimuli without interfering with the interaction with real objects (see left side of Fig. 3b). However, the perceptual effect of moving the haptic stimuli away from the expected contact location has yet to be studied. Another interesting insight regards the task completion time. The lowest average completion time $(47.5 \mathrm{~s}$, registered for condition CR) still seems quite high for the considered task. When we asked the subjects why they moved so slowly, three of them mentioned that the slight difference between their point of view and the one of the camera, shown on the screen, affected the intuitiveness of the interaction, slowing them down. This issue is then solved in the third experiment, where the Oculus Rift provided a compelling first-person view of the augmented environment.

In the third experiment, subjects are asked to balance a virtual sphere on a real cardboard. The haptic devices provide information about the weight of the virtual sphere rolling on the cardboard. This time, in all the considered metrics, providing cutaneous stimuli through the 3-RRS device (CF) outperformed providing cutaneous stimuli through the hRing device (CR). Since the cardboard rolled on top of the index finger pulp (see Fig. 7), only the 3-RRS device was able to correctly render all the directions of the augmented force. The hRing device was only able to provide forces normal to the cardboard and tangential stimuli in the mediallateral direction. This issue does also show in the subjective evaluation, where the condition providing feedback through the hRing (CR) showed similar appreciation than the one providing no force feedback at all (N). Finally, although the subjects still found it effective, 3 subjects out of 10 complained that the 3-RRS device was uncomfortable to wear while holding the cardboard.

\section{CONCLUSIONS AND FUtURE WORK}

This paper presents the experimental evaluation of two wearable haptic systems in three augmented reality applications. We employed a 3-RRS fingertip cutaneous device and a 2-DoF skin stretch cutaneous device. The 3-RRS device is composed of a rigid platform, driven by three servo motors, able to provide 3-DoF contact deformation stimuli to the user fingertip. The 2-DoF skin stretch cutaneous device is 
worn on the finger proximal phalanx and leaves the fingertip completely free. A moving belt, driven by two servo motors, provides skin stretch and normal stimuli to the user's skin.

We tested the two wearable interfaces in three AR experiments. In the first one, subjects had to write on a virtual board using a real chalk. The haptic devices provided the interaction forces between the chalk and the board. In the second experiment, subjects had to pick and place virtual and real objects mixed together. The haptic devices provided the interaction forces due to the weight of the virtual objects being picked up. In the third experiment, subjects had to balance a virtual sphere on a real cardboard. The haptic devices provided the interaction forces due to the weight of the virtual sphere rolling on the cardboard. In the first experiment, kinesthetic feedback provided by grounded haptic interfaces showed the best performance. However, since we seek for more compact and lightweight devices, we did not consider grounded haptic interfaces in the two other experiments. Amidst the other non-kinesthetic conditions, cutaneous feedback provided either through the hRing or the 3-RRS wearable devices outperformed sensory substitution via visual stimuli, which, in turn, outperformed providing no force feedback at all. The hRing device was the most preferred by the subjects, since it did not prevent them from using the fingertips to interact with the real environment.

In the next future, we will extend this evaluation to include more wearable devices, able to apply different cutaneous stimuli to different part of the body. Specifically, we will study more in detail the effect of vibrotactile stimuli in $\mathrm{AR}$, and we will consider haptic devices for the wrist and arm. Finally, we intend to investigate the practical translational aspects of these haptic-enabled augmented system, including its marketability and possibility of integration with available popular AR games and platforms.

\section{REFERENCES}

[1] I. E. Sutherland, "A head-mounted three dimensional display," in Proc. ACM December 9-11 fall joint computer conference, pp. 757-764, 1968.

[2] D. Etherington, "Techcruch: Pokémon GO estimated at over $75 \mathrm{~m}$ downloads worldwide," 2016. https://techcrunch.com/2016/07/ 25/pokemon-go-75m-downloads/.

[3] H. Taylor, "CNBC: Why apple is pouring money into virtual and augmented reality," 2016. http://www.cnbc.com/2016/08/10/ why-apple-is-pouring-money-into-virtual-and-augmented-reality. html.

[4] C. Newton, “The Verge: Facebook 2026," 2016. http://www. theverge.com/a/mark-zuckerberg-future-of-facebook.

[5] A. Liptak, "The Verge: Google reportedly working on headset that mixes augmented and virtual reality," 2016. http://www.theverge.com/2016/7/17/12209108/ google-headset-augmented-virtual-reality.

[6] N. Healey, "Cnet: Microsoft's mixed reality vision: 80 million devices by 2020," $2016 . \quad$ https://www.cnet.com/news/ microsofts-mixed-reality-vision-80-million-vr-and-ar-devices-by-202

[7] M. Solazzi, A. Frisoli, and M. Bergamasco, "Design of a cutaneous fingertip display for improving haptic exploration of virtual objects," in Proc. IEEE International Symposium on Robots and Human Interactive Communications, pp. 1-6, 2010.

[8] M. Gabardi, M. Solazzi, D. Leonardis, and A. Frisoli, "A new wearable fingertip haptic interface for the rendering of virtual shapes and surface features," in Proc. IEEE Haptics Symposium, pp. 140-146, 2016.

[9] D. Prattichizzo, F. Chinello, C. Pacchierotti, and M. Malvezzi, "Towards wearability in fingertip haptics: a 3-dof wearable device for cutaneous force feedback," IEEE Transactions on Haptics, vol. 6, no. 4, pp. 506-516, 2013.
[10] I. M. Koo, K. Jung, J. C. Koo, J.-D. Nam, Y. K. Lee, and H. R. Choi, "Development of soft-actuator-based wearable tactile display," IEEE Transactions on Robotics, vol. 24, no. 3, pp. 549-558, 2008.

[11] G. Frediani, D. Mazzei, D. E. De Rossi, and F. Carpi, "Wearable wireless tactile display for virtual interactions with soft bodies," Frontiers in bioengineering and biotechnology, vol. 2, 2014.

[12] D. Leonardis, M. Solazzi, I. Bortone, and A. Frisoli, "A wearable fingertip haptic device with 3 DoF asymmetric 3-rsr kinematics," in Proc. World Haptics Conference, pp. 388-393, 2015.

[13] D. Leonardis, M. Solazzi, I. Bortone, and A. Frisoli, "A 3-rsr haptic wearable device for rendering fingertip contact forces," IEEE Transactions on Haptics, 2016.

[14] A. Girard, M. Marchal, F. Gosselin, A. Chabrier, F. Louveau, and A. Lécuyer, "Haptip: Displaying haptic shear forces at the fingertips for multi-finger interaction in virtual environments," Frontiers in ICT, vol. 3, p. 6, 2016.

[15] I. Choi, E. W. Hawkes, D. L. Christensen, C. J. Ploch, and S. Follmer, "Wolverine: A wearable haptic interface for grasping in virtual reality," in Intelligent Robots and Systems (IROS), 2016 IEEE/RSJ International Conference on, pp. 986-993, 2016.

[16] L. Meli, S. Scheggi, C. Pacchierotti, and D. Prattichizzo, "Wearable haptics and hand tracking via an rgb-d camera for immersive tactile experiences," in Proc. ACM Special Interest Group on Computer Graphics and Interactive Techniques Conference, p. 56, 2014.

[17] H. Benko, C. Holz, M. Sinclair, and E. Ofek, "Normaltouch and texturetouch: High-fidelity $3 \mathrm{~d}$ haptic shape rendering on handheld virtual reality controllers," in Proceedings of the 29th Annual Symposium on User Interface Software and Technology, pp. 717$728,2016$.

[18] S. B. Schorr and A. Okamura, "Three-dimensional skin deformation as force substitution: Wearable device design and performance during haptic exploration of virtual environments," IEEE Transactions on Haptics, 2017.

[19] J. Arata, K. Ohmoto, R. Gassert, O. Lambercy, H. Fujimoto, and I. Wada, "A new hand exoskeleton device for rehabilitation using a three-layered sliding spring mechanism," in Proc. IEEE Int. Conf. on Robotics and Automation, pp. 3902-3907, 2013.

[20] C. J. Nycz, T. Bützer, O. Lambercy, J. Arata, G. S. Fischer, and R. Gassert, "Design and characterization of a lightweight and fully portable remote actuation system for use with a hand exoskeleton," IEEE Robotics and Automation Letters, vol. 1, no. 2, pp. 976-983, 2016.

[21] H. Uchiyama, M. A. Covington, and W. D. Potter, "Vibrotactile glove guidance for semi-autonomous wheelchair operations," in Proc. Annual Southeast Regional Conference, pp. 336-339, 2008.

[22] L. Hayes, "Vibrotactile feedback-assisted performance," in Proc. New Interfaces for Musical Expression, pp. 72-75, 2011.

[23] A. Hein and M. Brell, "contact-a vibrotactile display for computer aided surgery," in Proc. World Haptics, pp. 531-536, 2007.

[24] J. S. Zelek, S. Bromley, D. Asmar, and D. Thompson, "A haptic glove as a tactile-vision sensory substitution for wayfinding.," Journal of Visual Impairment \& Blindness, vol. 97, no. 10, pp. 1-24, 2003.

[25] E. Giannopoulos, A. Pomes, and M. Slater, "Touching the void: exploring virtual objects through a vibrotactile glove," The International Journal of Virtual Reality, vol. 11, pp. 19-24, 2012.

[26] J. Foottit, D. Brown, S. Marks, and A. M. Connor, "An intuitive tangible game controller," in Proc. Conference on Interactive Entertainment, pp. 1-7, 2014.

[27] A. Mazzoni and N. Bryan-Kinns, "Mood glove: A haptic wearable prototype system to enhance mood music in film," Entertainment Computing, 2016.

[28] C. Pacchierotti, S. Sinclair, M. Solazzi, A. Frisoli, V. Hayward, and D. Prattichizzo, "Wearable haptic systems for the fingertip and the hand: taxonomy, review, and perspectives," IEEE Transactions on Haptics. In Press, 2017.

[29] S. Scheggi, G. Salvietti, and D. Prattichizzo, "Shape and weight rendering for haptic augmented reality," in 19th International Symposium in Robot and Human Interactive Communication, pp. 44-49, 2010.

[30] F. Chinello, M. Malvezzi, C. Pacchierotti, and D. Prattichizzo, "Design and development of a 3RRS wearable fingertip cutaneous device," in Proc. IEEE/ASME International Conference on Advanced Intelligent Mechatronics, pp. 293-298, 2015.

[31] C. Pacchierotti, G. Salvietti, I. Hussain, L. Meli, and D. Prattichizzo, "The hRing: A wearable haptic device to avoid occlusions in hand tracking," in Proc. IEEE Haptics Symposium (HAPTICS), pp. 134-139, 2016. 
[32] K. H. Park, B. H. Kim, and S. Hirai, "Development of a softfingertip and its modeling based on force distribution," in Proc. of IEEE International Conference on Robotics and Automation, vol. 3, pp. 3169-3174, 2003.

[33] C. Pacchierotti, Cutaneous haptic feedback in robotic teleoperation. Springer Series on Touch and Haptic Systems, Springer International Publishing, 2015.

[34] K. Minamizawa, S. Fukamachi, H. Kajimoto, N. Kawakami, and S. Tachi, "Gravity grabber: wearable haptic display to present virtual mass sensation," in Proc. ACM Special Interest Group on Computer Graphics and Interactive Techniques Conference, pp. 8-es, 2007.

[35] K. Minamizawa, H. Kajimoto, N. Kawakami, and S. Tachi, “A wearable haptic display to present the gravity sensation-preliminary observations and device design," in Proc. of World Haptics, pp. 133138, 2007.

[36] C. B. Zilles and J. K. Salisbury, "A constraint-based god-object method for haptic display," in Proc. of IEEE/RSI International Conference on Intelligent Robots and Systems, vol. 3, pp. 146-151, 1995.

[37] L. Meli, C. Pacchierotti, and D. Prattichizzo, "Sensory subtraction in robot-assisted surgery: fingertip skin deformation feedback to ensure safety and improve transparency in bimanual haptic interaction," IEEE Transactions on Biomedical Engineering, vol. 61, no. 4, pp. 1318-1327, 2014.

[38] C. Pacchierotti, L. Meli, F. Chinello, M. Malvezzi, and D. Prattichizzo, "Cutaneous haptic feedback to ensure the stability of robotic teleoperation systems," International Journal of Robotics Research, vol. 34, no. 14, pp. 1773-1787, 2015.

[39] D. Prattichizzo, C. Pacchierotti, and G. Rosati, "Cutaneous force feedback as a sensory subtraction technique in haptics," IEEE Transactions on Haptics, vol. 5, no. 4, pp. 289-300, 2012.

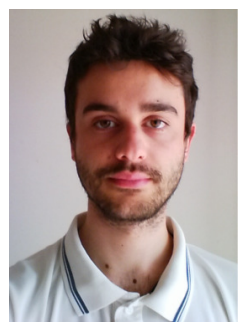

Maurizio Maisto received the B.S. degree in Electrical Engineering and the M.S. degree in Artificial Intelligence and Robotics from the Sapienza University of Rome, Italy, in 2014 and 2016 , respectively.

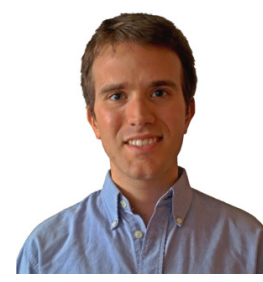

Claudio Pacchierotti (S'12, M'15) received the B.S., M.S., and Ph.D. degrees from the University of Siena, Italy in 2009, 2011, and 2014, respectively. He spent the first seven months of 2014 visiting the Penn Haptics Group at the University of Pennsylvania, Philadelphia, USA, which is part of the General Robotics, Automation, Sensing, and Perception (GRASP) Laboratory. He also visited the Dept. of Innovation in Mechanics and Management of the University of Padua and the Institute for Biomedical Technology and Technical Medicine (MIRA) of the University of Twente in 2013 and 2014, respectively. He received the 2014 EuroHaptics Best PhD Thesis Award for the best doctoral thesis in the field of haptics, and the 2015 Meritorious Service Award for his work as a Reviewer for the IEEE Transactions on Haptics. He has been a postdoctoral researcher at the Dept. of Advanced Robotics of the Italian Institute of Technology, Genova, Italy in 2015 and 2016. He is currently a CR2 researcher of the CNRS at Irisa and Inria Rennes Bretagne Atlantique, Rennes, France. His research deals with robotics and haptics, focusing on cutaneous force feedback techniques, wearable devices, and haptics for robotic surgery.

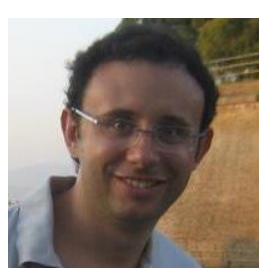

Francesco Chinello received the M.S. degree in computer engineering from the University of Siena, Italy, in 2010 and the Ph.D. in Automatic Control and Robotics in 2014 from the same University. He has been a postdoctoral researcher at the Dept. of Information Engineering and Mathematics of the University of Siena from 2014 to 2016. In 2016, he has also been a postdoctoral researcher at the Dept. of Advanced Robotics of the Italian Institute of Technology, Genova, Italy. $\mathrm{He}$ is currently an Assistant Professor at Aarhus University, Denmark. His interests include developing and testing robotic systems for research applications, with particular attention to force and position feedback systems.

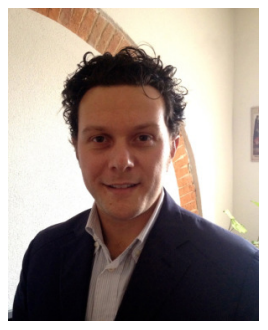

Gionata Salvietti (M'12) received the M.S. degree in Robotics and Automation and the Ph.D. degree in Information Engineering from the University of Siena, Siena, Italy, in 2009 and 2012, respectively. He was a post-doc researcher with the Istituto Italiano di Tecnologia from 2012 to 2015. He is currently Assistant Professor at Department of Information Engineering and Mathematics, University of Siena and Research Affiliate at Dept. of Advanced Robotics at Istituto Italiano di Tecnologia. From 2016, Associate Editor IEEE Robotics and Automation Letters. His research interests are telemanipulation, robotic and human grasping, haptics and assistive devices.

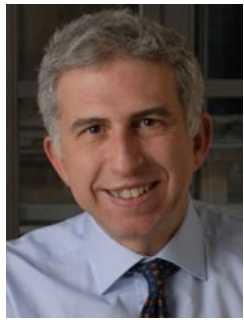

Alessandro De Luca (F'07) received his Ph.D. degree in Systems Engineering from Sapienza University of Rome, Rome, Italy, in 1987. He has been a Full Professor of robotics and automatic control with the Dipartimento di Ingegneria Informatica, Automatica e Gestionale, Sapienza University of Rome, since 2000. His research interests include robot control in general and in particular, flexible manipulators and physical human-robot interaction. Prof. De Luca has been an Editor of IEEE Transactions on Robotics and Automation (1998-2003), the first Editor-in-Chief of IEEE Transactions on Robotics (2004-2008), and General Chair and Program Chair of the IEEE International Conference on Robotics and Automation, respectively in 2007 and 2016. He has been the Coordinator of EU FP7 project SAPHARI (2011-2015).

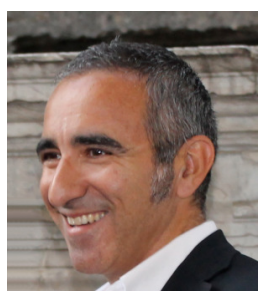

Domenico Prattichizzo (F'15) received the Ph.D. degree in Robotics and Automation from the University of Pisa in 1995. Since 2002 he is an Associate Professor of Robotics at the University of Siena and since 2009 he is a Scientific Consultant at Istituto Italiano di Tecnoloogia. In 1994, he was a Visiting Scientist at the MIT AI Lab. Since 2014, he is Associate Editor of Frontiers of Biomedical Robotics. From 2007 to 2013 he has been Associate Editor in Chief of the IEEE Transactions on Haptics. From 2003 to 2007, he has been Associate Editor of the IEEE Transactions on Robotics and IEEE Transactions on Control Systems Technologies. He has been Chair of the Italian Chapter of the IEEE RAS (2006-2010), awarded with the IEEE 2009 Chapter of the Year Award. Research interests are in haptics, grasping, visual servoing, mobile robotics and geometric control. $\mathrm{He}$ is currently the Coordinator of the IP collaborative project "WEARable HAPtics for Humans and Robots" (WEARHAP). 